\title{
MAXIMAL SCRAMBLED SETS FOR SIMPLE CHAOTIC FUNCTIONS
}

\author{
VÍCTOR JIMÉNEZ LÓPEZ
}

\begin{abstract}
This paper is a continuation of $[\mathbf{1}]$, where a explicit description of the scrambled sets of weakly unimodal functions of type $2^{\infty}$ was given. Its aim is to show that, for an appropriate non-trivial subset of the above family of functions, this description can be made in a much more effective and informative way.
\end{abstract}

\section{Introduction}

In [1] we found an explicit description of all scrambled sets of chaotic weakly unimodal functions of type $2^{\infty}$. Although as was shown there this description cannot be essentially simplified, it may not be very informative. The purpose of the present paper is to show that, if we restrict the family of functions under consideration in an appropriate way, our description provides us with an effective and detailed information about the structure of scrambled sets.

This paper has been devised to be self-contained, but a previous acquaintance with $[\mathbf{1}]$ would surely make its reading easier. We also refer to [1] for motivations, explanatory examples and some relevant bibliography. The rest of this introductory section is devoted to give an account of the main results from [1].

We begin with some necessary notations and definitions. Throughout the paper, closed intervals are possibly degenerate. The symbol $I$ will always denote a non-degenerate compact real interval. Given $A, B \subset I$, we shall write $A<B$ if $x<y$ for any $x \in A$ and $y \in B$. If $f$ is a function defined on $A$ and $B \subset A,\left.f\right|_{B}$ will denote the restriction of $f$ to $B$. $\mathrm{Cl} A$ and Card $A$ are the closure and the cardinality of $A$. For

This work has been partially supported by the DGICYT grant number PB91-0575. 
an arbitrary set $A, A^{\infty}$ and $A^{n}$ will denote the set of infinite sequences and finite sequences of length $n$ of elements from $A$. If $\alpha$ is one of such sequences, we shall denote its $i$-th term by $\alpha_{i}$. Sometimes, we shall describe abbreviately $\alpha$ using some smaller sequences. For instance, if $\beta=\beta_{1}, \beta_{2}, \ldots, \beta_{n}, \gamma=\gamma_{1}, \gamma_{2}, \ldots, \gamma_{m}$ and we write $\alpha=\beta, \gamma$, this means $\alpha=\beta_{1}, \beta_{2}, \ldots, \beta_{n}, \gamma_{1}, \gamma_{2}, \ldots, \gamma_{m}$. If $\alpha$ is infinite or its length is not less than $n$, we define $\left.\alpha\right|_{n}=\alpha_{1}, \alpha_{2}, \ldots, \alpha_{n}$. The shift map $\sigma: A^{\infty} \rightarrow A^{\infty}$ is defined by $\sigma(\alpha)=\alpha_{2}, \alpha_{3}, \alpha_{4}, \ldots$. $\mathbf{Z}$ is the set of integer numbers. The sequences $\mathbf{0}, \mathbf{1} \in \mathbf{Z}^{\infty}$ are defined by $\mathbf{0}_{i}=0$ and $\mathbf{1}_{i}=1$ for any $i$.

Let $f: I \rightarrow I$ be a continuous function. We denote $f^{0}=$ Id and define inductively $f^{n}=f^{n-1} \circ f$ for any $n \geq 1$. A point $p \in I$ is said to be periodic (of period $r$ ) if there is an $r \geq 1$ such that $f^{r}(p)=p$ and $f^{i}(p) \neq p$ for any $1 \leq i<r$. If $x \in I$ and there is periodic point $p$ with the property such that $\lim _{n \rightarrow \infty}\left|f^{n}(x)-f^{n}(p)\right|=0$, then $x$ is called asymptotically periodic. We say that $f$ is of type $2^{\infty}$ if it has periodic points of period $2^{r}$ for every $r \geq 0$, but no other periods. If $S$ is a subset of $I$ having at least two elements, and, for any $x, y \in S$, such that $x \neq y$, and any periodic point $p$ of $f$, we always have

(i) $\lim \sup _{n \rightarrow \infty}\left|f^{n}(x)-f^{n}(y)\right|>0$,

(ii) $\liminf _{n \rightarrow \infty}\left|f^{n}(x)-f^{n}(y)\right|=0$,

(iii) $\limsup _{n \rightarrow \infty}\left|f^{n}(x)-f^{n}(p)\right|>0$,

then $S$ is called a scrambled set of $f$, and we say that $f$ is chaotic (in the sense of Li and Yorke) (cf. [3], [2]). The important thing about chaos in the sense of $\mathrm{Li}$ and Yorke is that it is a valuable criterion to distinguish between "simple" and "complex" functions (from a dynamical point of view), as is clearly shown in [7]. A scrambled set of $f$ is maximal if is not properly included in another scrambled set. If $I=[a, b], f$ is non-constant, $f(a)=f(b) \in\{a, b\}$ and there is a $c \in(a ; b)$ such that $\left.f\right|_{[a ; c]}$ and $\left.f\right|_{[c ; b]}$ are (not necessary strictly) monotone then we say that $f$ is weakly unimodal. We shall denote by $U(I)(\operatorname{resp} . V(I))$ the set of weakly unimodal functions of type $2^{\infty}$ for which the corresponding point $c$ is an absolute maximum (resp. an absolute minimum). We also define $W(I)=U(I) \cup V(I)$. The set $W(I)$ is well known to contain both chaotic and nonchaotic functions (see for example [5]).

In order to find an explicit description of all maximal scrambled sets of chaotic functions from $W(I)$ we make use of a codification method (different from the standard Milnor and Thurston's kneading theory [4]). More precisely, we assign to any non-asymptotically periodic point a "code" (an infinite sequence of integer numbers) and study its dynamical properties in terms of the structure of its code. Observe that, since a scrambled set cannot contain any asymptotically periodic points, these 
last points are irrelevant for our purposes.

Our codification method is based in the idea of renormalization (see e.g. [8]). Let $f \in W(I)$ and let $K(f)$ denote the set of non-asymptotically periodic points of $f$. Then there exists a minimal closed interval $I_{1} \subset$ $I_{0}=I$ such that $f^{2}\left(I_{1}\right) \subset I_{1}$ and $\left.f^{2}\right|_{I_{1}} \in W\left(I_{1}\right)$. Let us denote $\Upsilon(f)=$ $\left.f^{2}\right|_{I_{1}}$, define inductively $f_{0}=f$ and $f_{i+1}=\Upsilon\left(f_{i}\right)$, and let $I_{i+1}$ be the interval playing the same role for $f_{i}$ as $I_{1}$ plays for $f$. It turns out that for any $x \in K(f)$ and $i \geq 0$ there is a minimal nonnegative integer $l(i, x)$ such that $x_{i}=f^{l(i, x)}(x) \in I_{n}$. Hence, we shall assign to any $x \in K(f)$ a sequence $\alpha=\Pi_{f}(x) \in \mathbf{Z}^{\infty}$ as follows. If $\alpha_{i}$ is the $i$-th term of the sequence $\alpha$, then $f_{i-1}$ maps $x_{i-1}$ into $I_{i}$ after $\left|\alpha_{i}\right|$ iterations and $f_{i-1}^{\left|\alpha_{i}\right|}\left(x_{i-1}\right)=x_{i}$. If $\alpha_{i}=0$, we have $x_{i-1}=x_{i}$ and then $x_{i-1} \in I_{i}$. Otherwise, $\alpha_{i}$ will be negative or positive depending on whether $x_{i-1}$ lies between $I_{i}$ and the fixed end point (for $f_{i-1}$ ) of $I_{i-1}$ or not.

Let us recall here some useful properties of the above sequences which we shall need later. For any $\alpha \in \mathbf{Z}^{\infty}$, define $K_{\alpha}(f)=\{x \in K(f)$ : $\left.\Pi_{f}(x)=\alpha\right\}$. It turns out that $K_{\alpha}(f)$ is a closed interval. Further, the function $f$ acts on the intervals $K_{\alpha}(f)$ as follows. If $\alpha \neq \mathbf{0}, j$ is the first integer such that $\alpha_{j} \neq 0$ and we define $\theta \in \mathbf{Z}^{\infty}$ by $\theta_{i}=1$ for any $i<j$, $\theta_{j}=1-\left|\alpha_{j}\right|$ and $\theta_{i}=\alpha_{i}$ otherwise, then

$$
f\left(K_{\alpha}(f)\right)=K_{\theta}(f) .
$$

Also,

$$
f\left(K_{\mathbf{0}}(f)\right) \subset K_{\mathbf{1}}(f) .
$$

On the other hand, assume $\beta \in \mathbf{Z}^{n}$. Then we define $K_{\beta}(f)=$ $\bigcup_{\alpha \in \mathbf{Z}^{\infty}:\left.\alpha\right|_{n}=\beta} K_{\alpha}(f)$. Now $K_{\beta}(f)$ is an open interval. The relative position of two intervals $K_{\beta}(f), K_{\gamma}(f), \beta, \gamma \in \mathbf{Z}^{n}$, can be described with the following order in $\mathbf{Z}^{n}$. If $\beta, \gamma \in \mathbf{Z}^{n}, \beta \neq \gamma$ and $k$ is the first integer such that $\beta_{k} \neq \gamma_{k}$, we say that $\beta<\gamma$ if either

$$
k+\operatorname{Card}\left\{1 \leq i<k: \beta_{i}>0\right\} \text { is odd and } \beta_{k}<\gamma_{k}
$$

or

$$
k+\operatorname{Card}\left\{1 \leq i<k: \beta_{i}>0\right\} \text { is even and } \gamma_{k}<\beta_{k} .
$$

Observe that in the case $n=1$ (when we identify $\mathbf{Z}^{1}$ and $\mathbf{Z}$ ), $<$ is the usual order in $\mathbf{Z}$. We have that

$$
\left.\beta<\gamma \text { if and only if } K_{\beta}(f)<K_{\gamma}(f) \text { [if } f \in U(I)\right]
$$


and

$$
\beta<\gamma \text { if and only if } K_{\gamma}(f)<K_{\beta}(f) \text { [if } f \in V(I) \text { ]. }
$$

If we want to describe all maximal scrambled sets of a function $f \in$ $W(I)$ we need to characterize explicitly (in terms of their codes) the points $x, y \in K(f)$ such that $\lim _{n \rightarrow \infty}\left|f^{n}(x)-f^{n}(y)\right|=0$ and $\liminf _{n \rightarrow \infty}\left|f^{n}(x)-f^{n}(y)\right|=0$ (more briefly, we shall write respectively in these cases $x \sim_{f}^{s} y$ and $x \sim_{f}^{i} y$ - or simply $x \sim^{s} y$ and $x \sim^{i} y$, if there is no confusion about $f$ ). Observe that $\sim^{s}$ is an equivalence relation in $K(f)$. Proposition 1.1 below describes explicitly $\sim^{i}$ and shows that it is an equivalence relation as well. We call every equivalence class for $\sim^{s}$ (resp. $\sim^{i}$ ) a $\sim_{f}^{s}$ class (resp. $\sim_{f}^{i}$ class) or simply a $\sim^{s}$-class (resp. $\sim^{i}$-class). Thus each maximal scrambled set of $f$ is characterized by the property of being included in some $\sim^{i}$-class $E$ and containing exactly one representative from every $\sim^{s}$-class included in $E$.

In what follows, if $\alpha \in \mathbf{Z}^{n}$ then we denote $\tau(\alpha)=\sum_{i=1}^{n}\left|\alpha_{i}\right| 2^{i-1}$.

Proposition 1.1. Let $f \in W(I), x, y \in K(f), \alpha=\Pi_{f}(x), \beta=$ $\Pi_{f}(y)$. Then $x \sim^{i} y$ if and only if $\tau\left(\left.\alpha\right|_{i}\right) \equiv \tau\left(\left.\beta\right|_{i}\right)\left(\bmod 2^{i}\right)$ for any $i$.

Unfortunately, the description of $\sim^{s}$ is much more complicated. First, we introduce in $K(f)$ for any $\gamma \in \mathbf{Z}^{\infty}$ the equivalence relation $\sim_{\gamma, f}^{s}$ (or simply $\sim_{\gamma}^{s}$ ) as follows: $x \sim_{\gamma}^{s} y$ if there is a $k$ large enough such that $K_{\gamma}(f) \not \subset\left(f^{n}(x) ; f^{n}(y)\right)$ for any $n>k$. (Here, $(u ; v)$ denotes respectively $(u, v),(v, u)$ or $\emptyset$ depending on whether $u<v, v<u$ or $u=v$.) It turns out that $\sim^{s}$ depends on some special $\sim_{\gamma}^{s}$. More precisely, let

$$
\mathcal{S}=\left\{\gamma \in\{0,1\}^{\infty}: \sigma^{i}(\gamma) \neq \mathbf{1} \text { for any } i\right\}
$$

Also, we say that $\gamma \in \mathcal{S}$ is an essential sequence of $f$ if $K_{\gamma}(f)$ is nondegenerate. Then $x \sim^{s} y$ if and only if $x \sim^{i} y$ and $x \sim_{\gamma}^{s} y$ for any essential sequence $\gamma$ of $f$. In some cases, two sequences $\gamma, \theta \in \mathcal{S}$ can induce the same equivalence relation, that is, $x \sim_{\gamma}^{s} y$ if and only if $x \sim_{\theta}^{s} y$ for any $x, y \in K(f)$. Then we say that $\gamma \sim_{e} \theta$ and call every equivalence class for $\sim_{e}$ a $\sim_{e}$-class. It can be checked that $\sim^{e}$ does not depend on the chosen function $f$. If $\mathcal{E}$ is a $\sim_{e}$-class containing some essential sequence of $f$ it will be called an essential class of $f$. Putting together all the above results, we get

Proposition 1.2. Let $f \in W(I), x, y \in K(f)$ and $\mathcal{E} \subset \mathcal{S}$ be a set containing exactly one representative from every essential class of $f$. Then $x \sim^{s} y$ if and only if $x \sim^{i} y$ and $x \sim_{\gamma}^{s} y$ for any $\gamma \in \mathcal{E}$. 
Thus, it remains to find only explicit descriptions of the equivalence relations $\sim_{e}$ and $\sim_{\gamma}^{s}$ for any $\gamma \in \mathcal{S}$. In the first case, this description is very simple:

Proposition 1.3. Let $\gamma, \theta \in \mathcal{S}$. Then $\gamma \sim_{e} \theta$ if and only if there is a $k$ such that $\sigma^{k}(\gamma)=\sigma^{k}(\theta)$.

Unfortunately, the second case is not so simple. We are forced to introduce some cumbersome notation. Let $\gamma \in \mathcal{S}$. Define the strictly increasing sequence $\left(o_{\gamma}(j)\right)_{j=1}^{\infty}$ (or simply $(o(j))_{j=1}^{\infty}$ if there is no confusion on $\gamma$ ) of the indexes $i$ with the property $\gamma_{i}=0$. Now we "cut" each $\alpha \in \mathbf{Z}^{\infty}$ into the blocks

$$
\alpha_{\gamma}^{j}=\alpha_{o(j-1)+1}, \alpha_{o(j-1)+2}, \ldots, \alpha_{o(j)}
$$

(or simply $\alpha^{j}$ ); here we mean $o(0)=0$. Next we introduce for any $\alpha \in \mathbf{Z}^{\infty}$ a sequence $\delta_{\gamma}^{\alpha} \in\{-1,0,1\}^{\infty}$, or simply $\delta^{\alpha}$, as follows (the $i$-th term of this sequence will be denoted by $\delta_{\gamma, i}^{\alpha}$ or $\left.\delta_{i}^{\alpha}\right)$. We put $\delta_{i}^{\alpha}=0$ if $i \neq o(j)$ for any $j \geq 1$. Now suppose that $i=o(j)$ for some $j \geq 1$. If $\tau\left(\alpha^{j}\right)<2^{i-o(j-1)-1}$ or $\tau\left(\alpha^{m}\right)<2^{o(m)-o(m-1)-1}$ for any $m>j$ then we define $\delta_{i}^{\alpha}=0$. Otherwise let $m>j$ be minimal such that $\tau\left(\alpha^{m}\right) \geq$ $2^{o(m)-o(m-1)-1}$ and define $\delta_{i}^{\alpha}=1$ if

$$
\alpha_{i}=0 \text { or } \alpha_{i+1}, \alpha_{i+2}, \ldots, \alpha_{o(m)}>\gamma_{i+1}, \gamma_{i+2}, \ldots, \gamma_{o(m)}
$$

and $\delta_{i}^{\alpha}=-1$ if

$$
\alpha_{i} \neq 0 \text { and } \alpha_{i+1}, \alpha_{i+2}, \ldots, \alpha_{o(m)}<\gamma_{i+1}, \gamma_{i+2}, \ldots, \gamma_{o(m)} \text {. }
$$

This finishes the definition of $\delta^{\alpha}$.

Moreover, let

$$
\mathcal{A}=\left\{\alpha \in \mathbf{Z}^{\infty}: \sigma^{i}(\alpha) \neq \mathbf{0} \text { for any } i\right\}
$$

and, for any $\alpha \in \mathbf{Z}^{\infty}$, define $\mathrm{n}(\alpha) \in \mathcal{A}$ by

$$
\mathrm{n}(\alpha)=\alpha
$$

if $\alpha \in \mathcal{A}$ and

$$
\mathrm{n}(\alpha)=2+\sup _{i} \tau\left(\left.\alpha\right|_{i}\right), 1,1,1,1,1,1, \ldots
$$

if $\alpha \in \mathbf{Z}^{\infty} \backslash \mathcal{A}$. Now we have 
Proposition 1.4. Let $f \in W(I), \gamma \in \mathcal{S}, x, y \in K, \alpha=\Pi_{f}(x)$, $\beta=\Pi_{f}(y)$. Put also $\theta=n(\alpha), \vartheta=n(\beta)$. Then $x \sim_{\gamma}^{s} y$ if and only if there is an $m$ such that $\tau\left(\left.\theta\right|_{o(m)}\right)=\tau\left(\left.\vartheta\right|_{o(m)}\right), \tau\left(\theta^{j}\right)=\tau\left(\vartheta^{j}\right)$ for any $j>m$ and $\delta_{i}^{\theta}=\delta_{i}^{\vartheta}$ for any $i>o(m)$.

We can summarize the above results in the following theorem:

Theorem 1.5. Let $f \in W(I)$ and $S \subset I$. Then the following statements hold.

(i) $f$ is chaotic if and only if it has some essential sequence.

(ii) Suppose $f$ is chaotic. Then $S$ is a maximal scrambled set of $f$ if and only if there is $a \sim^{i}$-class $E$ such that $S \subset E$ and $S$ contains exactly one representative from each of the $\sim^{s}$-classes included in E. Moreover, the equivalence relations $\sim^{i}$ and $\sim^{s}$ can be explicitly described (respectively, Proposition 1.1 and Propositions 1.2, 1.3 and 1.4).

Finally, let $\Phi:[0,1] \rightarrow[0,1]$ be defined by $\Phi(x)=\kappa x(1-x)$, where $\kappa \approx 3.5699456 \ldots$. As is well known, $\Phi \in W([0,1])$ (see e.g. [6]). It turns out that any function $f \in W(I)$ is topologically semiconjugate to $\Phi$, that is, there is a monotone onto function $g: I \rightarrow[0,1]$ such that $g \circ f=\Phi \circ g$. Although the function $g$ need not be unique, we shall fix one of them for any $f \in W(I)$, which will be denoted by $g_{f}$. Some relevant properties of the functions $\Phi$ and $g_{f}$ are:

$$
K_{\alpha}(\Phi) \text { is degenerate for any } \alpha \in \mathbf{Z}^{\infty} ;
$$

$$
\mathrm{Cl} K_{\beta}(\Phi)=\bigcup_{i \in \mathbf{Z}} \mathrm{Cl} K_{\beta, i}(\Phi)
$$

$\left(\right.$ and $\left.[0,1]=\bigcup_{i \in \mathbf{Z}} \mathrm{Cl} K_{i}(\Phi)\right)$;

$$
g_{f}\left(K_{\alpha}(f)\right)=K_{\alpha}(\Phi) \text { for any } \alpha \in \mathbf{Z}^{\infty} .
$$

This concludes the summary of the results from $[\mathbf{1}]$.

\section{Statement of the results}

One of the weak points of Theorem 1.5 is that, since if $\mathcal{E} \subset \mathcal{S}$ is a set containing exactly one representative from every essential class of $f$ then it may be infinite, we are forced to check $x \sim_{\gamma}^{s} y$ for infinitely many $\gamma$ in order to guarantee $x \sim^{s} y$. Of course, it would be nice to find a 
special $\gamma^{\prime} \in \mathcal{S}$ (maybe not necessarily holding $\gamma^{\prime} \sim_{e} \gamma$ for some $\gamma \in \mathcal{E}$ ) such that $x \sim^{s} y$ if and only if $x \sim_{\gamma^{\prime}}^{s} y$ for any $x \in K(f)$. If such a $\gamma^{\prime}$ exists, we shall say that $f$ is simple and call $\gamma^{\prime}$ a basic sequence of $f$. We remark that for any $\alpha \in \mathcal{S}$ there is a simple function $f$ having $\alpha$ as a basic sequence (see [1, Proposition 4]).

Not every function from $W(I)$ is simple. For instance, we give in [1] an example for which the corresponding set $\mathcal{E}$ is infinite and with the following additional property: if $\mathcal{E}^{\prime} \subset \mathcal{S}$ is a set intersecting each $\sim_{e^{-}}$ class in one point at most and, for any $x, y \in K(f), x \sim^{s} y$ if and only if $x \sim_{\gamma^{\prime}}^{s} y$ for any $\gamma^{\prime} \in \mathcal{E}^{\prime}$, then there is a bijection $\varphi: \mathcal{E} \rightarrow \mathcal{E}^{\prime}$ such that $\gamma \sim_{e} \varphi(\gamma)$ for any $\gamma \in \mathcal{E}$.

Hence, we begin by characterizing explicitly simple functions. First the notation. We denote

$$
\mathcal{S}_{0}=\left\{\gamma \in \mathcal{S}: \sigma^{k}(\gamma)=\mathbf{0} \text { for some } k\right\}
$$

and

$$
\begin{array}{r}
\mathcal{S}_{1}=\left\{\gamma \in \mathcal{S}: \text { there is a } k \text { such that if } i>k \text { and } \gamma_{i}=1\right. \\
\text { then } \left.\gamma_{i+1}=\gamma_{i+2}=0\right\} .
\end{array}
$$

If $\alpha, \beta \in \mathcal{A}$ and $\gamma \in \mathcal{S}$, we say that $\alpha \sim_{\gamma}^{s} \beta$ if for a fixed $f \in W(I)$ and $x, y \in K(f)$ with $\alpha=\mathrm{n}(\Pi(x)), \beta=\mathrm{n}(\Pi(y))$, then we have $x \sim_{\gamma, f}^{s} y$. Notice that by Proposition 1.4 this definition does not depend on the chosen $f, x$ and $y$. Finally, if $\gamma, \theta \in \mathcal{S}$, we say that $\gamma \triangleleft \theta$ if $\alpha \sim_{\gamma}^{s} \beta$ implies $\alpha \sim_{\theta}^{s} \beta$ for any $\alpha, \beta \in \mathcal{A}$. The characterizacion of simple functions depends esentially on the property $\triangleleft$. Thus we give first an explicit description of $\triangleleft$ :

Theorem 2.1. Let $\gamma \in \mathcal{S}$. Then the following statements hold.

(i) Suppose that $\gamma \in \mathcal{S}_{0}$. Then $\gamma \triangleleft \theta$ for any $\theta \in \mathcal{S}$.

(ii) Suppose that $\gamma \in \mathcal{S}_{1}$. Then $\gamma \triangleleft \theta$ if and only if either $\gamma \sim_{e} \theta$ or $\gamma \chi_{e} \theta$ and there is a number $k$ such that, if $(j(n))_{n=1}^{\infty}$ denotes the strictly increasing sequence of the integers $j$ with the property $\gamma_{\theta}^{j} \neq \theta_{\theta}^{j}$ and $n>k$, then we have:

(a) Either

$$
\begin{aligned}
\gamma_{\theta}^{j(n)} & =0,0, \ldots, 0,1,0,0, \\
\gamma_{\theta}^{j(n)} & =0,0, \ldots, 0,1,0,
\end{aligned}
$$

or

$$
\gamma_{\theta}^{j(n)}=0,0, \ldots, 0
$$


(b) If $j(n)<j<r<j(n+1)$ are consecutive integers such that $\gamma_{\theta}^{j}=\gamma_{\theta}^{r}=1,0$ then $r-j$ is even. Also, if there is a maximal number $j(n)<r<j(n+1)$ with the property $\gamma_{\theta}^{r}=1,0$ then $j(n+1)-r$ is even.

(c) If

$$
\gamma_{\theta}^{j(n)}=0,0, \ldots, 0,1,0,0
$$

(resp.

$$
\left.\gamma_{\theta}^{j(n)}=0,0, \ldots, 0,1,0,\right),
$$

then $j(n+1)-j(n)$ is odd (resp. even).

(iii) Suppose that $\gamma \in \mathcal{S} \backslash\left(\mathcal{S}_{0} \cup \mathcal{S}_{1}\right)$. Then $\gamma \triangleleft \theta$ if and only if $\gamma \sim_{e} \theta$.

Let us describe briefly the conditions in the theorem in the case $\gamma \in \mathcal{S}_{1}$ [recall that $\gamma \in \mathcal{S}_{1}$ if for any $j$ large enough we have either $\gamma_{\gamma}^{j}=0$ or $\gamma_{\gamma}^{j}=1,0$ (and in this last case we must have $\gamma_{\gamma}^{j+1}=0$ )]. Apart from the trivial case $\gamma \sim_{e} \theta$, if we want to get $\gamma \triangleleft \theta$ then we must have necessarily either

$$
\begin{aligned}
\gamma_{\theta}^{j(n)} & =0,0, \ldots, 0,1,0,0 \\
\gamma_{\theta}^{j(n)} & =0,0, \ldots, 0,1,0,
\end{aligned}
$$

or

$$
\gamma_{\theta}^{j(n)}=0,0, \ldots, 0,0
$$

for any $n$ large enough (in particular, $\gamma_{\theta}^{j(n)}$ can have one "1" at most). Further, if $j(n+1)>j(n)+1$ then the sequence of blocks

$$
\gamma_{\theta}^{j(n)+1}, \gamma_{\theta}^{j(n)+2}, \ldots, \gamma_{\theta}^{j(n+1)-1}
$$

(which coincides with a sequence

$$
\gamma_{\gamma}^{l+1}, \gamma_{\gamma}^{l+2}, \ldots, \gamma_{\gamma}^{l+j(n+1)-j(n)-2}
$$

for some appropriate $l$ ) has the following structure. In the case

$$
\gamma_{\theta}^{j(n)}=0,0, \ldots, 0,1,0
$$

we find first a maximal sequence of zeros of odd length, while if

$$
\gamma_{\theta}^{j(n)}=0,0, \ldots, 0,1,0,0
$$


then we have at the beginning a (possibly empty) maximal sequence of zeros of even length; in the case

$$
\gamma_{\theta}^{j(n)}=0,0, \ldots, 0,0
$$

the initial number of zeros is arbitrary. From now on, the structure is always the same: the first block 1,0 may appear, then an odd number of zeros follow, again a block of the type 1,0 , an odd number of zeros and so on. If the sequence

$$
\gamma_{\theta}^{j(n)+1}, \gamma_{\theta}^{j(n)+2}, \ldots, \gamma_{\theta}^{j(n+1)-1}
$$

contains some block of the type 1,0 , it always finishes with an odd number of zeros.

Some possible cuts of two sequences $\gamma, \theta$ in the above conditions could be

$$
\begin{aligned}
& \gamma=\ldots ; 0,0 ; 0 ; 1,0 ; 0 ; 0 ; 0 ; 0,1,0 ; 0 ; 1,0,0 ; 0,0,0,0 ; 0 ; 0 ; 0,0 ; \ldots \\
& \theta=\ldots ; 1,0 ; 0 ; 1,0 ; 0 ; 0 ; 0 ; 1,1,0 ; 0 ; 1,1,0 ; 1,1,1,0 ; 0 ; 0 ; 1,0 ; \ldots
\end{aligned}
$$

where the semicolons mark the end of every block $\gamma_{\theta}^{j}$ or $\theta_{\theta}^{j}$. Observe that for any given $\gamma \in \mathcal{S}_{1}$ there is always a $\theta$ with $\gamma \triangleleft \theta$ and $\gamma \chi_{e} \theta$. In fact, if $k$ is large enough so that $i>k$ and $\gamma_{i}=1$ imply $\gamma_{i+1}=\gamma_{i+2}=0$, then it is sufficient to define $\theta$ by $\theta_{i}=0$ if $i-2>k$ and $\gamma_{i-2}=1$, and $\theta_{i}=1$ otherwise.

Now we can give the promised explicit characterization of simple functions:

Theorem 2.2. Let $f \in W(I)$ and let $\mathcal{E} \subset \mathcal{S}$ be a set containing exactly one representative from each essential class of $f$. Then the following statements are equivalent.

(i) $f$ is simple.

(ii) There is a $\gamma^{\prime} \in \mathcal{E}$ such that $\gamma^{\prime} \triangleleft \theta$ for any $\theta \in \mathcal{E}$.

But even for simple functions, things are not that clear. The description of the property $\sim^{s}$ (which is now equivalent to that of $\sim_{\gamma}^{s}$ for a given basic sequence $\gamma$ ) involves so many conditions that, for example, it is difficult at first sight to distinguish the cases when the $\sim^{s}$-class containing a given point $x$ consists of a countable or uncountable number of intervals $K_{\alpha}(f)$. Then we shall devote the rest of the paper to analyze in full detail the topological structure of the $\sim^{i}$ and $\sim^{s}$ classes for this type of functions. From a topological point of view, things may be 
distorted because some of the intervals $K_{\alpha}(f)$ may be non-degenerate. Hence, we shall better use the semiconjugacy $g_{f}$ (cf. (7) and (9) above) and study the images of these sets via $g_{f}$. The question of which of the intervals $K_{\alpha}(f)$ must be non-degenerate for a given $f \in W(I)$ was precisely answered in [1, Proposition 4].

Our first results work in fact for any function from $W(I)$ :

Proposition 2.3. Let $f \in W(I)$ and let $E$ be $a \sim^{i}$-class of $f$. Then $g_{f}(\mathrm{Cl} E)$ is a Cantor set and $0,1 \in g_{f}(\mathrm{Cl} E)$. Further, let $\left\{\left(a_{n}, b_{n}\right)\right\}_{n}$ be the family of connected components of $[0,1] \backslash g_{f}(\mathrm{Cl} E)$. Then $g_{f}(E)=$ $g_{f}(\mathrm{Cl} E) \backslash\left(\{0,1\} \cup \bigcup_{n}\left\{a_{n}, b_{n}\right\}\right)$.

Proposition 2.4. Let $f \in W(I)$, let $E$ be $a \sim^{i}$-class of $f$ and let $F \subset E$ be $a \sim^{s}$-class of $f$. Then $g_{f}(F)$ is dense in $g_{f}(E)$.

To precise the topological structure of $\sim^{s}$-classes is a harder task. We say that a set $A \subset[0,1]$ is small (resp. big) if it is infinite and countable (resp. if it is an infinite countable union of pairwise disjoint Cantor sets). It will turn out that, after applying $g_{f}$, each $\sim^{s}$ class is either a big or a small set. Moreover, big and small $\sim^{s}$ classes can be explicitly characterized. With this aim we introduce the following definition.

Let $\gamma \in \mathcal{S}_{1}$ and $\alpha \in \mathbf{Z}^{\infty}$. We say that $\alpha$ is exceptional (for $\gamma$ ) if $\sigma^{i}\left(\delta_{\gamma}^{\alpha}\right) \neq \mathbf{0}$ for any $i$ and, if $(j(n))_{n=1}^{\infty}$ denotes the strictly increasing sequence of the numbers $j$ with the property $\delta_{\gamma, o_{\gamma}(j)}^{\alpha} \neq 0$, then there is a number $k$ such that the following properties hold for any $n>k$.

(i) If $\gamma_{\gamma}^{j(n)}=1,0$ then $\alpha_{\gamma}^{j(n)}=1,1$. If $j(n)<j<j(n+1)$ and $\gamma_{\gamma}^{j(n)}=1,0$ then $\alpha_{\gamma}^{j(n)}=1,0$.

(ii) If $j(n) \leq j<r \leq j(n+1)$ are consecutive numbers with the property $\gamma_{\gamma}^{j}=\gamma_{\gamma}^{r}=1,0$ then $r-j$ is even.

(iii) Suppose that $\gamma_{\gamma}^{j(n+1)}=0$ and there is a minimal $j(n) \leq j<$ $j(n+1)$ with the property $\gamma_{\gamma}^{j}=1,0$. If $\alpha_{o_{\gamma}(j(n+1))}<0$ (resp. $\left.\alpha_{o_{\gamma}(j(n+1))}>0\right)$ then $j(n+1)-j$ is odd (resp. even).

Hence, if $j$ is large enough then either $\alpha_{\gamma}^{j}=1,0, \alpha_{\gamma}^{j}=1,1$ or $\alpha_{\gamma}^{j}=l$ for some integer $l$. Further, let $j(n)$ and $j(n+1)$ be consecutive numbers with the property $\delta_{\gamma, o_{\gamma}(j(n))}^{\alpha} \neq 0 \neq \delta_{\gamma, o_{\gamma}(j(n+1))}^{\alpha}$ (that is, the blocks $\alpha_{\gamma}^{j(n)}, \alpha_{\gamma}^{j(n+1)}$ equal either 1,1 or $l$ for some integer $l \neq 0$ ). If $\alpha_{\gamma}^{j(n)}=l$, $\alpha_{\gamma}^{j(n+1)}=l^{\prime}$ for some $l, l^{\prime} \in \mathbf{Z} \backslash\{0\}$ and no blocks of the type 1,0 are located between $\alpha_{\gamma}^{j(n)}$ and $\alpha_{\gamma}^{j(n+1)}$, then no further restrictions are necessary. On the other hand, if $j(n) \leq j<r \leq j(n+1)$ and $\alpha_{\gamma}^{j}, \alpha_{\gamma}^{r}$ are two consecutive blocks of length 2 then they must be separated by 
an odd number of zeros. Finally, if $\alpha_{\gamma}^{j(n+1)}=l$ for some $l \in \mathbf{Z} \backslash\{0\}$ and there is a minimal $j(n) \leq j<j(n+1)$ such that $\alpha_{\gamma}^{j}$ has length 2 , then we must have

$$
\alpha_{\gamma}^{j+1}, \alpha_{\gamma}^{j+2}, \ldots, \alpha_{\gamma}^{j(n+1)}<\gamma_{\gamma}^{j+1}, \gamma_{\gamma}^{j+2}, \ldots, \gamma_{\gamma}^{j(n+1)} .
$$

A possible cut of such a sequence $\alpha$ after $\alpha_{o_{\gamma}(j(k))}$ could for example be

$$
\begin{aligned}
\ldots ; 2 ; 0 ; 3 ; 1,1 ; 0 ; 7 ; 1,0 ; 0 ; 0 ; 0 ; 0 ; 0 ; 1,0 ; 0 ; 8 \\
\quad 1,0 ; 0 ; 1,1 ; 0 ; 0 ; 0 ; 1,0 ; 0 ; 1,1 ; 0 ; 0 ; 0 ; 1,0 ; 0 ; 0 ; 0 ; 1,0 ;-7 \ldots,
\end{aligned}
$$

the semicolons delimiting the blocks $\alpha_{\gamma}^{j}$. Now we have

Theorem 2.5. Let $f \in W(I)$ be a simple function and let $\gamma$ be a basic sequence of $f$. Let $x \in K(f), \alpha=n\left(\Pi_{f}(x)\right)$ and let $F$ be the $\sim^{s}$-class of $f$ containing $x$. Then the following statements hold.

(i) If $\gamma \in \mathcal{S}_{0}$ then $g_{f}(F)$ is small.

(ii) If $\gamma \in \mathcal{S}_{1}$ and $\alpha$ is exceptional for $\gamma$ then $g_{f}(F)$ is small; otherwise $g_{f}(F)$ is big.

(iii) If $\gamma \in \mathcal{S} \backslash\left(\mathcal{S}_{0} \cup \mathcal{S}_{1}\right)$ then $g_{f}(F)$ is big.

We give the proofs of the above results in the next section. Until the end of this paper, Proposition 1.4 and (3), (4) will be repeatedly used with no further references.

\section{Proofs}

Proof of Theorem 2.1: The statement (i) is immediate, because if $\gamma \in$ $\mathcal{S}_{0}$ and $\alpha, \beta \in \mathcal{A}$ are such that $\alpha \sim_{\gamma}^{s} \beta$ then there is a $k$ such that $\tau\left(\left.\alpha\right|_{k}\right)=\tau(\beta \mid k)$ and $\alpha_{i}=\beta_{i}$ for any $i>k$. Thus $\alpha \sim_{\theta}^{s} \beta$ for any $\theta \in \mathcal{S}$.

Now we prove simultaneously (ii) and (iii). For this purpose it is sufficient to show the following

Claim: If $\gamma, \theta \in \mathcal{S} \backslash \mathcal{S}_{0}$ are given and $\gamma \chi_{e} \theta$, then $\gamma \triangleleft \theta$ if and only if $\gamma \in \mathcal{S}_{1}$ and the conditions (a), (b) and (c) in (ii) are satisfied (with $(j(n))_{n=1}^{\infty}$ defined as there).

In order to simplify the notation, we shall always write $o(j)$ instead of $o_{\theta}(j), \delta^{\alpha}$ instead of $\delta_{\theta}^{\alpha}$ and $\alpha^{j}$ instead of $\alpha_{\theta}^{j}$ for any $j \geq 1$ and $\alpha \in \mathbf{Z}^{\infty}$.

We begin by proving the "only if" part of the Claim. We shall proceed in (seven) steps. Each of them consists of finding a number $k_{j}$ such 
that a certain property of the sequences $\gamma, \theta$ holds for any $n>k_{j}$ (here $n$ refers to the indexes of the sequence $\left.(j(n))_{n=1}^{\infty}\right)$. When proving the " $j$-th" step, we shall implicitly use the results from the previous ones. For this reason, we shall always assume that the numbers $k_{j}$ have been chosen in such a way that $k_{j+1}>k_{j}$ for any $j$.

Step 1. There is a number $k_{1}$ with the following property: if $n>k_{1}$ then there is an $m$ such that $o(n)=o_{\gamma}(m)$.

Suppose the contrary. Then we can find a sequence of numbers $(r(s))_{s=1}^{\infty}$ with the properties $\gamma_{r(s)}=1, \theta_{r(s)}=0$ and $r(s+1)-r(s)>1$ for any $s$. Let $\alpha, \beta \in \mathbf{Z}^{\infty}$ be defined $\alpha_{i}, \alpha_{i+1}=3,1, \beta_{i}, \beta_{i+1}=1,2$ if $i=r(s)$ for some $s$ and $\alpha_{i}=\beta_{i}=1$ otherwise. It is easy to check that $\alpha \sim_{\gamma}^{s} \beta$. On the other hand, if for a given $s$ we find $j$ with the property $o(j)=r(s)$ then we clearly have $\tau\left(\alpha^{j}\right) \neq \tau\left(\beta^{j}\right)$. Hence, $\alpha \chi_{\theta}^{s} \beta$, a contradiction.

Step 2. There is a number $k_{2}$ with the following property: if $n>k_{2}$ and $r$ is such that $o(j(n)-1)<o_{\gamma}(r)<o(j(n))$ and $\gamma_{\gamma}^{r} \neq 0$, then $o_{\gamma}(r+1)=o(j(n))$.

Assume not. Then there are (strictly increasing) sequences $(r(s))_{s=1}^{\infty}$, $(n(s))_{s=1}^{\infty}\left(\right.$ with $n(s)>k_{1}$ for any $\left.s\right)$ such that $o(j(n(s))-1)<o_{\gamma}(r(s))<$ $o(j(n(s)))$ and $\gamma_{\gamma}^{r(s)} \neq 0$ but $o_{\gamma}(r(s)+1)<o(j(n(s)))$ for any $s$. Define $\alpha, \beta \in \mathbf{Z}^{\infty}$ by $\alpha_{i-1}, \alpha_{i}=3,0, \beta_{i-1}, \beta_{i}=1,1$ if $i=o_{\gamma}(r(s))$ for some $s$, $\alpha_{i}=\beta_{i}=-1$ if $i=o_{\gamma}(r(s)+1)+1$ for some $s$, and $\alpha_{i}=\beta_{i}=1$ otherwise. Observe that, for any $s, \delta_{o(j(n(s))-1)}^{\alpha}=1, \delta_{o(j(n(s))-1)}^{\beta}=-1$ because $\alpha_{o(j(n(s))-1)} \neq 0 \neq \beta_{o(j(n(s))-1)}$ and $\beta^{j(n(s))}<\theta^{j(n(s))}<\alpha^{j(n(s))}$. On the other hand, we have $\alpha_{o_{\gamma}(r(s))}=0$ and $\beta_{\gamma}^{r(s)+1}>\gamma_{\gamma}^{r(s)+1}$, so $\delta_{\gamma, o_{\gamma}(r(s))}^{\alpha}=$ $\delta_{\gamma, o_{\gamma}(r(s))}^{\beta}=1$. From this we get easily that $\alpha \sim_{\gamma}^{s} \beta$ and $\alpha \chi_{\theta}^{s} \beta$, which is impossible.

Step 3. There is a number $k_{3}$ with the following property: if $n>k_{3}$ and $r$ is such that $o(j(n)-1)<o_{\gamma}(r)<o(j(n))$ and $\gamma_{\gamma}^{r} \neq 0$, then $\gamma_{\gamma}^{r+1}=0$.

On the contrary, find sequences $(r(s))_{s=1}^{\infty},(n(s))_{s=1}^{\infty}\left(\right.$ with $n(s)>k_{2}$ for any $s)$ such that $o(j(n(s))-1)<o_{\gamma}(r(s))<o(j(n(s)))$ and $\gamma_{\gamma}^{r(s)} \neq 0$ but $\gamma_{\gamma}^{r(s)+1} \neq 0$ or any $s$. Additionally, we can always assume $n(s+$ $1)-n(s)>1$. Also, $m(s)$ will denote the first integer greater than $o(j(n(s)+1)-1)$ with the property $\gamma_{m(s)}=0$.

Further, it is not restrictive to assume that one of the following two possibilities must occur: either $j(n(s)+1)-j(n(s))$ is odd for any $s$ or $j(n(s)+1)-j(n(s))$ is even for any $s$. In the first case, define $\alpha, \beta$ by $\alpha_{i-1}, \alpha_{i}=3,0, \beta_{i-1}, \beta_{i}=1,1$ if $i=o_{\gamma}(r(s)+1)$ for some $s, \alpha^{j}=\beta^{j}=\gamma^{j}$ 
for any $j(n(s))<j<j(n(s)+1)$ and any $s, \alpha_{i}=\beta_{i}=-1$ if $i=m(s)+1$ for some $s$ and $\alpha_{i}=\beta_{i}=1$ otherwise. Recall that $n(s+1)-n(s)>1$ for any $s$, and then $\alpha$ and $\beta$ are well defined. Let $u$ be such that $o_{\gamma}(u-1)=$ $o(j(n(s)+1)-1)$. Since $\beta_{\gamma}^{u}>\gamma_{\gamma}^{u}$ and $j(n(s)+1)-j(n(s))$ is odd, we get that

$$
\beta^{j(n(s))+1}, \ldots, \beta^{j(n(s)+1)-1}, \beta_{\gamma}^{u}>\gamma^{j(n(s))+1}, \ldots, \gamma^{j(n(s)+1)-1}, \beta_{\gamma}^{u} .
$$

Thus, $\delta_{\gamma, o_{\gamma}(r(s)+1)}^{\alpha}=\delta_{\gamma, o_{\gamma}(r(s)+1)}^{\beta}=1$ for any $s$. Now it is easy to check that $\alpha \sim_{\gamma}^{s} \beta$. However, we have $\beta^{j(n(s)+1)}<\gamma^{j(n(s)+1)}$ and then $\delta_{o(j(n(s)))}^{\alpha}=1, \delta_{o(j(n(s)))}^{\beta}=-1$. This means that $\alpha \chi_{\theta}^{s} \beta$.

The case when $j(n(s)+1)-j(n(s))$ is even for any $s$ is similar. Now we define $\alpha, \beta$ by $\alpha_{i-1}, \alpha_{i}=3,0, \beta_{i-1}, \beta_{i}=1,1$ if $i=o_{\gamma}(r(s))$ for some $s, \alpha_{\gamma}^{r(s)+1}=\beta_{\gamma}^{r(s)+1}=\gamma_{\gamma}^{r(s)+1}, \alpha^{j}=\beta^{j}=\gamma^{j}$ for any $j(n(s))<j<$ $j(n(s)+1)$ and any $s, \alpha_{i}=\beta_{i}=-1$ if $i=m(s)+1$ for some $s$, and $\alpha_{i}=\beta_{i}=1$ otherwise. We have $\delta_{\gamma, o_{\gamma}(r(s))}^{\alpha}=\delta_{\gamma, o_{\gamma}(r(s))}^{\beta}=1$ for any $s$ (we use that $j(n(s)+1)-j(n(s))$ is even) and so $\alpha \sim_{\gamma}^{s} \beta$. On the other hand, notice that $\alpha_{o(j(n(s))-1)} \neq 0 \neq \beta_{o(j(n(s))-1)}$. Further, $\alpha^{j(n(s))}>\theta^{j(n(s))}$ and, since $\beta^{j(n(s)+1)}<\gamma^{j(n(s)+1)}$, we also have

$$
\begin{aligned}
\beta^{j(n(s))}, \beta^{j(n(s))+1} & \ldots, \beta^{j(n(s)+1)-1}, \beta^{j(n(s)+1)} \\
& <\gamma^{j(n(s))}, \gamma^{j(n(s))+1}, \ldots, \gamma^{j(n(s)+1)-1}, \gamma^{j(n(s)+1)}
\end{aligned}
$$

Thus $\delta_{o(j(n(s))-1)}^{\alpha}=1$ and $\delta_{o(j(n(s)))}^{\beta}=-1$ for any $s$, which implies $\alpha \chi_{\theta}^{s} \beta$.

Step 4. There is a number $k_{4}$ with the following property: if $n>k_{4}$ and $r$ is such that $o(j(n)-1)<o_{\gamma}(r) \leq o(j(n))$ and $\gamma_{\gamma}^{r} \neq 0$, then $\gamma_{\gamma}^{r}=1,0$.

In the opposite case, find some sequences $(r(s))_{s=1}^{\infty},(n(s))_{s=1}^{\infty}$ (with $n(s)>k_{3}$ for any $\left.s\right)$ such that $o(j(n(s))-1)<o_{\gamma}(r(s)) \leq o(j(n(s)))$ and $\gamma_{\gamma}^{r(s)} \neq 0, \gamma_{\gamma}^{r(s)} \neq 1,0$. As in Step 3, we assume $n(s+1)-n(s)>1$ for any $s$. Also, let $m(s)>o(j(n(s)+1)-1)$ be the first integer with the property $\gamma_{m(s)}=0$.

We can suppose that either $o_{\gamma}(r(s))<o(j(n(s)))$ for any $s$ or $o_{\gamma}(r(s))=o(j(n(s)))$ for any $s$. If the first possibility occurs, define $\alpha, \beta$ by

$$
\begin{gathered}
\alpha_{i-2}, \alpha_{i-1}, \alpha_{i}, \alpha_{i+1}=3,0,0,-1 \\
\beta_{i-2}, \beta_{i-1}, \beta_{i}, \beta_{i+1}=1,1,0,-1
\end{gathered}
$$


if $i=o_{\gamma}(r(s))$ for some $s$ and $\alpha_{i}=\beta_{i}=1$ otherwise. Then we have $\alpha_{o_{\gamma}(r(s)-1)} \neq 0 \neq \beta_{o_{\gamma}(r(s)-1)}$. Further,

$$
\alpha_{\gamma}^{r(s)}, \alpha_{\gamma}^{r(s)+1}>\gamma_{\gamma}^{r(s)}, \gamma_{\gamma}^{r(s)+1}
$$

and

$$
\beta_{\gamma}^{r(s)}, \beta_{\gamma}^{r(s)+1}>\gamma_{\gamma}^{r(s)}, \gamma_{\gamma}^{r(s)+1},
$$

so $\delta_{\gamma, o_{\gamma}(r(s)-1)}^{\alpha}=\delta_{\gamma, o_{\gamma}(r(s)-1)}^{\beta}=1$ for any $s$. Hence, $\alpha \sim_{\gamma}^{s} \beta$. On the other hand, we clearly have $\delta_{o(j(n(s))-1)}^{\alpha}=1$ and $\delta_{o(j(n(s))-1)}^{\beta}=-1$ for any $s$, and then $\alpha \chi_{\theta}^{s} \beta$.

If $o_{\gamma}(r(s))=o(j(n(s)))$ for any $s$ then we can further assume that either $j(n(s)+1)-j(n(s))$ is odd for any $s$ or $j(n(s)+1)-j(n(s))$ is even for any $s$. Now define $\alpha, \beta$ as follows. In the first case, put $\alpha_{i-1}, \alpha_{i}=3,0, \beta_{i-1}, \beta_{i}=1,1$ if $i=o_{\gamma}(r(s))$ for some $s, \alpha^{j}=\beta^{j}=\gamma^{j}$ for any $j(n(s))<j<j(n(s)+1)$ and any $s, \alpha_{i}=\beta_{i}=-1$ if $i=m(s)+1$ for some $s$ and $\alpha_{i}=\beta_{i}=1$ otherwise. If $j(n(s)+1)-j(n(s))$ is even for any $s$, put $\alpha_{i-2}, \alpha_{i-1}, \alpha_{i}=3,0,0, \beta_{i-2}, \beta_{i-1}, \beta_{i}=1,1,0$ if $i=o_{\gamma}(r(s))$ for some $s, \alpha^{j}=\beta^{j}=\gamma^{j}$ for any $j(n(s))<j<j(n(s)+1)$ and any $s$, $\alpha_{i}=\beta_{i}=-1$ if $i=m(s)+1$ for some $s$ and $\alpha_{i}=\beta_{i}=1$ otherwise. As in the analogous cases in Step 3, we can check that $\alpha$ and $\beta$ are well defined, $\alpha \sim_{\gamma}^{s} \beta$ and $\alpha \not_{\theta}^{s} \beta$.

Step 5. There is a number $k_{5}$ with the following property: if $n>k_{5}$ and $j(n)<j<r<j(n+1)$ are consecutive integers with $\gamma^{j} \neq 0 \neq \gamma^{r}$ then $r-j$ is even; moreover, if there is a maximal $j(n)<r<j(n+1)$ such that $\gamma^{r} \neq 0$ then $j(n+1)-r$ is even.

If the statement from Step 5 does not hold, it is possible to find some sequences $(r(s))_{s=1}^{\infty},(n(s))_{s=1}^{\infty}$ (with $n(s)>k_{4}$ for any $s$ ) with $j(n(s))<$ $r(s)<j(n(s)+1)$ and such that $\gamma^{r(s)} \neq 0$ and $j(n(s)+1)-r(s)$ is odd. As usual, let $m(s)>o(j(n(s)+1)-1)$ be the first integer such that $\gamma_{m(s)}=0$. Let us define $\alpha, \beta$ by $\alpha_{i-1}, \alpha_{i}=3,0, \beta_{i-1}, \beta_{i}=1,1$ if $i=o(r(s))$ for some $s, \alpha^{j}=\beta^{j}=\gamma^{j}$ for any $r(s)<j<j(n(s)+1)$ and any $s, \alpha_{i}=\beta_{i}=-1$ if $i=m(s)+1$ for some $s$ and $\alpha_{i}=\beta_{i}=1$ otherwise. As in the case when $j(n(s)+1)-j(n(s))$ is odd in Step 3, we obtain that $\alpha \sim_{\gamma}^{s} \beta$ and $\alpha \not_{\theta}^{s} \beta$.

Step 6. There is a number $k_{6}$ with the following property: if $n>k_{6}$ and $j(n)<j<j(n+1)$ then either $\gamma^{j}=0$ or $\gamma^{j}=1,0$.

On the contrary, find $(r(s))_{s=1}^{\infty},(n(s))_{s=1}^{\infty}\left(\right.$ with $n(s)>k_{5}$ for any $s$ ) such that $j(n(s))<r(s)<j(n(s)+1)$ and $\gamma^{r(s)} \neq 0, \gamma^{r(s)} \neq 1,0$, and define the numbers $m(s)$ as usual. Let $\alpha, \beta$ be defined by $\alpha_{i-2}, \alpha_{i-1}, \alpha_{i}=$ $3,0,0, \beta_{i-2}, \beta_{i-1}, \beta_{i}=1,1,0$ if $i=o(r(s))$ for some $s, \alpha^{j}=\beta^{j}=\gamma^{j}$ for 
any $r(s)<j<j(n(s)+1)$ and any $s, \alpha_{i}=\beta_{i}=-1$ if $i=m(s)+1$ for some $s$ and $\alpha_{i}=\beta_{i}=1$ otherwise. Then we have $\alpha \sim_{\gamma}^{s} \beta$ and $\alpha \mathcal{\psi}_{\theta}^{s} \beta$ as in the case when $j(n(s)+1)-j(n(s))$ is even in Step 3 .

Step \%. There is a number $k_{7}$ with the following property: if $n>k_{7}$ and $\gamma^{j(n)}=0,0, \ldots, 0,1,0,0$ (resp. $\left.\gamma^{j(n)}=0,0, \ldots, 0,1,0\right)$ then $j(n+$ $1)-j(n)$ is odd (resp. even).

Suppose not. Find $(r(s))_{s=1}^{\infty},(n(s))_{s=1}^{\infty}$ (with $n(s)>k_{6}$ for any $s$ ) such that $\gamma^{j(n)}=0,0, \ldots, 0,1,0,0\left(\right.$ resp. $\left.\gamma^{j(n)}=0,0, \ldots, 0,1,0\right)$ and $j(n(s)+1)-j(n(s))$ is even (resp. odd) for any $s$. Define the numbers $m(s)$ as above and assume here $n(s+1)-n(s)>1$ for any $s$. Now define $\alpha, \beta$ by $\alpha_{i-2}, \alpha_{i-1}, \alpha_{i}=3,0,0, \beta_{i-2}, \beta_{i-1}, \beta_{i}=1,1,0$ (resp. $\alpha_{i-1}, \alpha_{i}=$ $\left.3,0, \beta_{i-1}, \beta_{i}=1,1\right)$ if $i=o(n(s))$ for some $s, \alpha^{j}=\beta^{j}=\gamma^{j}$ for any $j(n(s))<j<j(n(s)+1)$ and any $s, \alpha_{i}=\beta_{i}=-1$ if $i=m(s)+1$ for some $s$ and $\alpha_{i}=\beta_{i}=1$ otherwise. As in Step $3, \alpha \sim_{\gamma}^{s} \beta$ and $\alpha \chi_{\theta}^{s} \beta$.

From Steps 1-7, the "only if" part of the Claim follows easily.

Let us prove now the "if" part of the Claim. Reasoning by contradiction, assume the existence of two sequences $\alpha, \beta \in \mathcal{A}$ with $\alpha \sim_{\gamma}^{s} \beta$ and $\alpha \chi_{\theta}^{s} \beta$. Clearly, we can assume that there are some numbers $r>j(k)$ and $s$ [with $k$ defined as in (ii)] such that $o_{\gamma}(s)=o(r), \tau\left(\alpha_{\gamma}^{j}\right)=\tau\left(\beta_{\gamma}^{j}\right)$ for any $j \geq s$,

$$
0 \neq \delta_{o(r)}^{\alpha} \neq \delta_{o(r)}^{\beta} \neq 0
$$

and

$$
\delta_{\gamma, i}^{\alpha}=\delta_{\gamma, i}^{\beta} \text { for any } i \geq o_{\gamma}(s)
$$

Observe that the case $\alpha_{o(r)}=\beta_{o(r)}=0$ is impossible by (10). Now assume that $\alpha_{o(r)}=0, \beta_{o(r)} \neq 0$. Find an appropriate $n$ such that $j(n) \leq r<j(n+1)$. Since $\delta_{o(r)}^{\alpha}=1$, we get $\delta_{o(r)}^{\beta}=-1$ by (10). Further, $\alpha_{o(r)} \neq \beta_{o(r)}$ and $\tau\left(\alpha_{\gamma}^{j}\right)=\tau\left(\beta_{\gamma}^{j}\right)$ for any $j \geq s$ imply that $\theta^{r} \neq 0$ (and $\left.\gamma_{\gamma}^{s}=1,0\right)$. Also, (10) and (11) force $\beta^{j}=\gamma^{j}$ for any $r<j<j(n+1)$. Hence, $\theta^{j(n+1)} \leq \beta^{j(n+1)}$. On the other hand, $\delta_{\gamma, o_{\gamma}(s)}^{\alpha}=\delta_{\gamma, o_{\gamma}(s)}^{\beta}=1$ by (11) and so $\beta_{\gamma}^{u} \leq \gamma_{\gamma}^{u}$, where $u$ is such that $o_{\gamma}(u-1)=o(j(n+1)-1)$. Since $\gamma_{\gamma}^{u}<\theta_{\gamma}^{u}$, we get a contradiction.

Since the case $\alpha_{o(r)} \neq 0, \beta_{o(r)}=0$ is analogous to the previous one, it remains to consider only the possibility $\alpha_{o(r)} \neq 0 \neq \beta_{o(r)}$. Let $t>o(r)$ be the first integer such that $\alpha_{t} \neq \theta_{t}$ or $\beta_{t} \neq \theta_{t}$ (let us say $\alpha_{t} \neq \theta_{t}$ ). We have several alternatives.

Case 1: There is an $n$ such that $o(j(n)-1)<t \leq o(j(n))$. 
Now three possibilities appear. Firstly suppose that $\gamma^{j(n)}=$ $0,0, \ldots, 0,0$. By (11), $\alpha_{o(j(n)-1)+1}=\beta_{o(j(n)-1)+1}$. Then, $\alpha_{o(j(n)-1)+1}=$ $\beta_{o(j(n)-1)+1}=1$ by (10). Similarly, we deduce $\alpha_{i}=\beta_{i}=1$ for any $o(j(n)-1)<i<o(j(n))$ and then $t=o(j(n))$. This, together with (11), gives $\alpha_{o(j(n)}=\beta_{o(j(n)} \neq 0$, contradicting $(10)$.

Next, suppose that $\gamma^{j(n)}=0,0, \ldots, 0,1,0,0$. Then we deduce similarly $\alpha_{i}=\beta_{i}=1$ for any $o(j(n)-1)<i \leq o(j(n))-3$. Further, if we want (10) and (11) to be simultaneously fulfilled, one of the sequences $\alpha_{o(j(n))-2}, \alpha_{o(j(n))-1}$ and $\beta_{o(j(n))-2}, \beta_{o(j(n))-1}$ (for example say $\left.\beta_{o(j(n))-2}, \beta_{o(j(n))-1}\right)$ must equal 1,0 or 1,1 . If $\beta_{o(j(n))-2}, \beta_{o(j(n))-1}=$ 1,0 we must have either $\alpha_{o(j(n))-2}, \alpha_{o(j(n))-1}=1,0$ or $\alpha_{o(j(n))-2}, \alpha_{o(j(n))-1}=-1,0$, in both cases contradicting (10). Then $\beta_{o(j(n))-2}, \beta_{o(j(n))-1}=1,1$ and now (11) forces $\alpha_{o(j(n))-2}, \alpha_{o(j(n))-1}=$ 3,0 (so $t=o(j(n))-2$ ). (We are omitting the other analogous possibility, $\alpha_{o(j(n))-2}, \alpha_{o(j(n))-1}=1,1, \beta_{o(j(n))-2}, \beta_{o(j(n))-1}=3,0$.) Now, $\beta_{o(j(n))} \leq 0$ by $(10)$ and $\beta_{o(j(n))} \geq 0$ by (11). Hence, $\alpha_{o(j(n))}=\beta_{o(j(n))}=$ 0 . Similarly, if $j(n+1)-j(n)>1$ then (10) implies

$$
\gamma^{j(n)+1}, \ldots, \gamma^{j(n+1)-1} \leq \beta^{j(n)+1}, \ldots, \beta^{j(n+1)-1},
$$

while by (11)

$$
\beta^{j(n)+1}, \ldots, \beta^{j(n+1)-1} \leq \gamma^{j(n)+1}, \ldots, \gamma^{j(n+1)-1} .
$$

Then,

$$
\beta^{j(n)+1}, \ldots, \beta^{j(n+1)-1}=\gamma^{j(n)+1}, \ldots, \gamma^{j(n+1)-1} .
$$

Finally, let $u$ be such that $o_{\gamma}(u-1)=o(j(n+1)-1)$. Then $\theta^{j(n+1)} \leq$ $\beta^{j(n+1)}$ by (10) and $\beta_{\gamma}^{u} \leq \gamma_{\gamma}^{u}$ by (11), which is impossible.

Finally, suppose $\gamma^{j(n)}=0,0, \ldots, 0,1,0$. Reasoning in a similar way we get $\alpha_{i}=\beta_{i}=1$ for any $o(j(n)-1)<i \leq o(j(n))-2$, while now for example $\alpha_{o(j(n))-1}, \alpha_{o(j(n))}=-1,0, \beta_{o(j(n))-1}, \beta_{o(j(n))}=1,0$ and $t=o(j(n))-1$ (we omit the similar case $\alpha_{o(j(n))-1}, \alpha_{o(j(n))}=1,0$ and $\left.\beta_{o(j(n))-1}, \beta_{o(j(n))}=-1,0\right)$. Also,

$$
\beta^{j(n)+1}, \ldots, \beta^{j(n+1)-1}=\gamma^{j(n)+1}, \ldots, \gamma^{j(n+1)-1}
$$

if $j(n+1)-j(n)>1$. If $u$ is such that $o_{\gamma}(u-1)=o(j(n+1)-1)$, we obtain again the contradiction $\theta^{j(n+1)} \leq \beta^{j(n+1)}$ and $\beta_{\gamma}^{u} \leq \gamma_{\gamma}^{u}$.

Case 2: There is an $n$ such that $o(j-1)<t \leq o(j)$ for some $j(n)<$ $j<j(n+1)$. 
In this case it is not difficult to see that $\gamma^{j}=1,0$. Then $\alpha^{j}=-1,0$, $\beta^{j}=1,0$ (or $\left.\alpha^{j}=1,0, \beta^{j}=-1,0\right)$ and we get a contradiction by reasoning similarly to the last paragraph from Case 1 .

From this, the "if part" of the Claim follows. Theorem 2.1 is proved.

Proof of Theorem 2.2: Due to Theorem 1.5, (ii) implies (i). To prove that (i) implies (ii) we must show that if $f$ is simple and $\gamma$ is a basic sequence of $f$ then there is a $\gamma^{\prime} \in \mathcal{E}$ such that $\gamma \sim_{e} \gamma^{\prime}$.

Suppose the contrary. Denote $\mathcal{E}=\left\{{ }^{n} \gamma\right\}_{n=1}^{r}$, where $1 \leq r \leq \infty$. Since $\gamma \triangleleft{ }^{n} \gamma$ for any $n$, there is a sequence $(k(n))_{n=1}^{r}$ such that if $i>k(n)$ and $\gamma_{i}=1$ then ${ }^{n} \gamma_{i}=1$ (see Step 1 in the proof of Theorem 2.1). Let $(i(n))_{n=1}^{\infty}$ be a strictly increasing sequence such that $k(n)<i(n)$ for any $n$ and with the property that there is an $i(n)<i<i(n+1)$ with $\gamma_{i}=0$ and ${ }^{l} \gamma_{i}=1$ for any $n$ and any $1 \leq l \leq n$. We can also assume that $\gamma_{i(n)}=0$ for any $n$.

Define now $\alpha, \beta \in \mathbf{Z}^{\infty}$ in the following way. If $i=i(n)$ for some $n$, put $\alpha_{i}=2, \beta_{i}=-2$. Otherwise, put $\alpha_{i}=\beta_{i}=\gamma_{i}$. We have that $\alpha \sim_{n_{\gamma}}^{s} \beta$ for any $n$. To prove it, fix $n$ and take $k$ large enough so that $o_{n} \gamma(k)>k(n)$. Let $j>k$. If $o_{n} \gamma(j)=i(m)$ for some $m$, we have that either

$$
\alpha_{i(m)+1}, \ldots, \alpha_{i(m+1)}<{ }^{n} \gamma_{i(m)+1}, \ldots,{ }^{n} \gamma_{i(m+1)}
$$

and

$$
\beta_{i(m)+1}, \ldots, \beta_{i(m+1)}<{ }^{n} \gamma_{i(m)+1}, \ldots,{ }^{n} \gamma_{i(m+1)},
$$

or

$$
\alpha_{i(m)+1}, \ldots, \alpha_{i(m+1)}>{ }^{n} \gamma_{i(m)+1}, \ldots,{ }^{n} \gamma_{i(m+1)}
$$

and

$$
\beta_{i(m)+1}, \ldots, \beta_{i(m+1)}>{ }^{n} \gamma_{i(m)+1}, \ldots,{ }^{n} \gamma_{i(m+1)} .
$$

Then we get respectively $\delta_{n_{\gamma, o_{n}(j)}}^{\alpha}=\delta_{n_{\gamma, o_{\gamma}(j)}}^{\beta}=-1$ or $\delta_{n_{\gamma, o_{n}(j)}}^{\alpha}=$ $\delta_{n_{\gamma, o n_{\gamma}(j)}^{\beta}}^{\beta}=1$. If $o_{n_{\gamma}}(j) \neq i(m)$ for any $m$ then we have $\alpha_{o_{n_{\gamma}}(j)}=$ $\beta_{o n_{\gamma}(j)}=0$. Hence, either $\delta_{n_{\gamma, o n_{\gamma}(j)}}^{\alpha}=\delta_{n_{\gamma, o n_{\gamma}(j)}}^{\beta}=0$ or $\delta_{n_{\gamma, o n_{\gamma}(j)}}^{\alpha}=$ $\delta_{n_{\gamma, o n_{\gamma}(j)}}^{\beta}=1$. So, in any case $\delta_{n_{\gamma, o_{\gamma}}(j)}^{\alpha}=\delta_{n_{\gamma, o n_{\gamma}(j)}}^{\beta}$ for any $j>k$ and then $\alpha \sim_{n_{\gamma}}^{s} \beta$ as we desired to show.

On the other hand, we obviously have $\alpha \not_{\gamma}^{s} \beta$; a contradiction.

Proof of Proposition 2.3: By (9), it is not restrictive to suppose $f=\Phi$, $g_{f}=$ Id. Fix $\alpha \in \Pi_{f}(E)$ and define

$$
\Lambda_{n}=\left\{\beta \in \mathbf{Z}^{n}: \tau\left(\left.\alpha\right|_{i}\right) \equiv \tau\left(\left.\beta\right|_{i}\right) \quad\left(\bmod 2^{i}\right) \text { for any } 1 \leq i \leq n\right\}
$$


for any $n \geq 1$. Also, write $U_{n}=\bigcup_{\beta \in \Lambda_{n}} K_{\beta}(f)$ and let $A_{n}$ denote the set containing the points 0,1 and all the end points of the intervals $K_{\beta}(f)$, $\beta \in \bigcup_{i=1}^{n} \Lambda_{i}$. Finally, put $A=\bigcup_{n=1}^{\infty} A_{n}$.

By Proposition 1.1, $E=\bigcap_{n=1}^{\infty} U_{n}$. It is easy to check that $\Lambda_{1}$ is either the set of even integers or the set of odd integers, and if $\beta \in \Lambda_{k}$ for some $k$ then $\Lambda_{k+1} \cap\{\beta, j: j \in \mathbf{Z}\}$ is either $\{\beta, 2 r: r \in \mathbf{Z}\}$ or $\{\beta, 2 r+1: r \in \mathbf{Z}\}$. Hence, (8) implies $\mathrm{Cl} U_{n}=U_{n} \cup A_{n}$. Since $\mathrm{Cl} E \subset \mathrm{Cl} U_{n}$ for any $n$, we get $\mathrm{Cl} E \subset E \cup A$. On the other hand we clearly have $E \cup A \subset \mathrm{Cl} E$ again by (8), so $\mathrm{Cl} E=E \cup A$. Since $\mathrm{Cl} E$ has empty interior and has no isolated points [use (7)], $\mathrm{Cl} E$ is a Cantor set. Furthermore, observe that $A \backslash\{0,1\}$ is the set of all the end points of the connected components of $[0,1] \backslash \mathrm{Cl} E$. This completes the proof.

Proof of Proposition 2.4: We can again assume $f=\Phi, g_{f}=\mathrm{Id}$. Let $G=\{y \in F: \Pi(y)=\mathrm{n}(\Pi(y))\}$. Since $g(G)$ is dense in $g(E)$ [see the proof of Proposition 2.3 and use $(7)$ ], we only need to prove that $g(F)$ is dense in $g(G)$. Let $x \in G$ and put $\Pi_{f}(x)=\alpha$. It suffices, fixed $k$ large enough, to find $\beta \in \mathbf{Z}^{\infty}$ different from $\alpha$ with the properties $\left.\alpha\right|_{k}=\left.\beta\right|_{k}$ and $\tau\left(\left.\alpha\right|_{l}\right)=\tau\left(\left.\beta\right|_{l}\right), \sigma^{l}(\alpha)=\sigma^{l}(\beta)$ for some appropriate $l>k$. Indeed, we could choose then $y \in K(f)$ with $\Pi_{f}(y)=\beta$ : $y$ would be very close to $x$ by (7) and $y \in F$ because $f^{\tau\left(\left.\alpha\right|_{l}\right)}(x)=f^{\tau\left(\left.\alpha\right|_{l}\right)}(y)$ [by (1), (2)]. But it is very easy to construct such a $\beta$. Just find $l>k+1$ such that $\alpha_{l} \neq 0$, say $\tau\left(\left.\alpha\right|_{l}\right)-\tau\left(\left.\alpha\right|_{k}\right)=m 2^{k}, m \geq 1$, and define $\beta$ by $\beta_{k+1}=m, \beta_{i}=0$ if $k+2 \leq i \leq l$, and $\beta_{i}=\alpha_{i}$ otherwise.

The proof of Theorem 2.5 follows after a sequence of lemmas. The simple chaotic function $f$ and the basic sequence $\gamma$ of $f$ will remain fixed. In the notation, we shall omit some explicit references to $f$ and $\gamma$. So, we shall put $g$ instead of $g_{f}$ and $\Pi(y)$ instead of $\Pi_{f}(y)$ for any $y \in K(f)$. Also, we write $o(j)$ instead of $o_{\gamma}(j), \delta^{\beta}$ instead of $\delta_{\gamma}^{\beta}$ and $\beta^{j}$ instead of $\beta_{\gamma}^{j}$ for any $j \geq 1$ and any $\beta \in \mathbf{Z}^{\infty}$. Additionally, we fix a $\sim^{s}$-class $F$ of $f$, choose $x \in F$ and denote $\alpha=\mathrm{n}(\Pi(x))$.

Lemma 3.1. If $\gamma \in \mathcal{S}_{0}$ then $g(F)$ is small.

Proof: Recall that $\alpha \sim_{\gamma}^{s} \beta$ if and only if there is a $k$ such that $\tau\left(\left.\alpha\right|_{k}\right)=$ $\tau(\beta \mid k)$ and $\alpha_{i}=\beta_{i}$ for any $i>k$. From this, the lemma follows [use also (7) and (9)].

Lemma 3.2. If $\gamma \in \mathcal{S}_{0}$ and $\alpha$ is exceptional then $g(F)$ is small.

Proof: It suffices to prove that $\mathrm{n}(\Pi(F))$ is countable. Let $\beta \in \mathrm{n}(\Pi(F))$. We can assume that if $k$ and $(j(n))_{n=1}^{\infty}$ are the numbers from the definition of exceptional sequence for $\alpha$, then $\tau\left(\left.\alpha\right|_{o(j(k))}\right)=\tau\left(\left.\beta\right|_{o(j(k))}\right)$, 
$\tau\left(\alpha^{j}\right)=\tau\left(\beta^{j}\right)$ for any $j>j(k)$ and $\delta_{i}^{\alpha}=\delta_{i}^{\beta}$ for any $i>o(j(k))$. Fix $r>k$. It is sufficient to show that $\alpha^{j}=\beta^{j}$ for any $j(r)<j \leq j(r+1)$. We distinguish several possibilities.

Case 1. $\gamma^{j(r)}=1,0$.

Here it is easy to check that $\delta_{o(j(r))}^{\alpha}=-1$, so $\delta_{o(j(r))}^{\beta}=-1$ as well. In particular, $\beta_{o(j(r)} \neq 0$. Suppose $\alpha^{j} \neq \beta^{j}$ for some $j(r)<j \leq j(r+1)$. If $j<j(r+1)$ then $\beta^{j}=-1,0$ and so $\delta_{o(j(r))}^{\beta}=1$, a contradiction. If $j=j(r+1)$ then $\delta_{o(j(r))}^{\beta}=-1$ forces $\beta^{j}=3,0$, which is impossible because we can prove that $\beta_{o(j(r+1))} \neq 0$ as before.

Case 2. $\gamma^{j(r)}=0, \gamma^{j(r+1)}=1,0$.

If $j(r)<j \leq j(r+1)$ is such that $\alpha^{j} \neq \beta^{j}$ then $\delta_{o(j(r))}^{\beta}=\delta_{o(j(r))}^{\alpha} \neq 0$ clearly implies $j=j(r+1)$ and $\beta^{j(r+1)}=3,0$. But this possibility was discarded in Case 1 , so a contradiction arises.

Case 3. $\gamma^{j(r)}=\gamma^{j(r+1)}=0$.

If $\gamma^{j}=0$ for any $j(r)<j \leq j(r+1)$ then we have obviously $\alpha^{j}=\beta^{j}=$ 0 for any $j(r)<j<j(r+1)$ and both $\alpha_{o(j(r+1)}$ and $\beta_{o(j(r+1)}$ have the same sign because $\delta_{o(j(r))}^{\alpha}=\delta_{o(j(r))}^{\beta}$. Hence, $\alpha_{o(j(r+1)}=\beta_{o(j(r+1)}$ and we are done. On the other hand, if there is a minimal if $j(r)<l<j(r+1)$ with the property $\gamma^{l}=1,0$ and $j(r)<j \leq j(r+1)$ is such that $\alpha^{j} \neq \beta^{j}$ then we must have $j \geq l$ and $\beta^{j}=-1,0$. Thus $\beta^{j}, \beta^{j+1}, \ldots, \beta^{j(n+1)}<\gamma^{j}, \gamma^{j+1}, \ldots, \gamma^{j(n+1)}$, in contradiction with $\alpha^{j}, \alpha^{j+1}, \ldots, \alpha^{j(n+1)}>\gamma^{j}, \gamma^{j+1}, \ldots, \gamma^{j(n+1)}$ and $\delta_{o(j(r))}^{\alpha}=\delta_{o(j(r))}^{\beta}$.

This proves the lemma.

Lemma 3.3. If either $\gamma \in \mathcal{S} \backslash\left(\mathcal{S}_{0} \cup \mathcal{S}_{1}\right)$ or $\gamma \in \mathcal{S}_{0} \cup \mathcal{S}_{1}$ and $\alpha$ is not exceptional then $g(F)$ is big.

Proof: Denote $G=\{y \in F: \Pi(y)=\mathrm{n}(\Pi(y))\}$. For any $m \geq 1$ and any $\beta \in \mathbf{Z}^{o(m)}$ such that $\tau\left(\left.\alpha\right|_{o(m)}\right)=\tau(\beta)$, let $G_{m, \beta}$ be the set of points $y$ from $G$ such that $\left.\Pi(y)\right|_{o(m)}=\beta$ and $m$ is minimal with the properties $\tau\left(\left.\Pi(y)\right|_{o(m)}\right)=\tau\left(\left.\alpha\right|_{o(m)}\right), \tau\left(\Pi(y)^{j}\right)=\tau\left(\alpha^{j}\right)$ for any $j>m$ and $\delta_{i}^{\Pi(y)}=\delta_{i}^{\alpha}$ for any $i>o(m)$.

Obviously, $G$ is the union set of all the sets $G_{m, \beta}$ defined above and observe that some sets $G_{m, \beta}$ may be empty. Also, each $G_{m, \beta}$ is closed. To prove it, let $\left(y_{n}\right)_{n=1}^{\infty}$ be a sequence of points from $G_{m, \beta}$ converging to some $y \in I$. Clearly, we can find $\theta \in \mathcal{A}$ and strictly increasing sequences $(n(r))_{r=1}^{\infty},(i(r))_{r=1}^{\infty}$ such that $\left.\Pi\left(y_{n(r)}\right)\right|_{i(r)}=\left.\theta\right|_{i(r)}$ for any $r$. Thus $\Pi(y)=\theta$ [use (5) and (6)] and $y \in G_{m, \beta}$. 
Suppose that $\gamma$ and $\alpha$ are in the conditions of the lemma and redenote the family of the nonempty sets $G_{m, \beta}$ by $\left\{G^{s}\right\}_{s}$. We desire to show that $g(F)$ is big. For this purpose we claim that it is sufficient to show that each $g\left(G^{s}\right)$ has no isolated points. Indeed, in this case $g\left(G^{s}\right)$ is a Cantor set by Proposition 2.3. Further, notice that $g(F) \backslash g(G)$ is countable (possibly empty). If $u \in g(F) \backslash g(G)$ then we can find a sequence $\left(G^{s(n)}\right)_{n=1}^{\infty}$ such that the distances between $g\left(G^{s(n)}\right)$ and $u$ tend to zero (because $g(G)$ is dense in $g(F)$, see the proof of Proposition 2.4). Since the diameters of the sets $g\left(G^{s(n)}\right)$ tend to zero as well, $\{u\} \cup$ $\bigcup_{n=1}^{\infty} g\left(G^{s(n)}\right)$ is a Cantor set (again by Proposition 2.3). Similarly, if $u^{\prime} \neq u$ belongs to $g(F) \backslash g(G)$ then there is a family $\left\{G^{s^{\prime}(n)}\right\}_{n=1}^{\infty}$ (with the sets $G^{s^{\prime}(n)}$ disjoint from the sets $\left.G^{s(n)}\right)$ such that $\left\{u^{\prime}\right\} \cup \bigcup_{n=1}^{\infty} g\left(G^{s^{\prime}(n)}\right)$ is a Cantor set. Proceeding in this way, we obtain that $g(F)$ is big (observe that $g(F)$ cannot be a finite union of Cantor sets by Proposition 2.3).

Hence, fixed $G^{s}$, we shall show that $g\left(G^{s}\right)$ has no isolated points. Let $\beta \in \Pi\left(G^{s}\right)$. Clearly, it is sufficient to find for any given $k$ a sequence $\theta \in \mathbf{Z}^{\infty}$ different from $\beta$ with the properties $\left.\beta\right|_{k}=\left.\theta\right|_{k}, \tau\left(\beta^{j}\right)=\tau\left(\theta^{j}\right)$ for any $j \geq 1$ and $\delta_{i}^{\beta}=\delta_{i}^{\theta}$ for any $i \geq 1$. If $\sigma^{i}\left(\delta^{\beta}\right)=\mathbf{0}$ for some $i$ then such a sequence $\theta$ clearly exists, so we shall assume that $\sigma^{i}\left(\delta^{\beta}\right) \neq \mathbf{0}$ for any $i$ and $(j(n))_{n=1}^{\infty}$ will denote the strictly increasing sequence of the numbers $j$ with the property $\delta_{o(j)}^{\beta} \neq 0$. Fix a number $l$ with $j(l)>k$. Since $\alpha$ is not exceptional, $\beta$ cannot be exceptional either (otherwise $\sigma^{i}(\alpha)=\sigma^{i}(\beta)$ for some $i$, see the proof of Lemma 3.2). Then it is not difficult to check that one of the following cases must necessarily occur and that the sequence $\theta$ defined in each of them has the desired properties. (When considering Case $n$, we shall implicitly assume that neither of the previous cases simultaneously holds.)

Case 1. There are some $r>l$ and $j(r)<s<j(r+1)$ such that $\beta^{s} \neq 0, \beta^{s} \neq 1,0$.

We shall assume that $s$ is minimal with the above property. Now there are two possibilities:

Subcase 1.a. $\beta^{s}=\gamma^{s}$. Since $\beta^{s} \neq 1,0$, its length $u$ must be greater than 2. For example suppose that $\delta_{o(j(r))}^{\beta}=-1$ (in the case $\delta_{o(j(r))}^{\beta}=1$ we can proceed in a similar way). Put $t=\tau\left(\beta^{s}\right)>1$. Because of the minimality of $s$, we have that either

$$
\beta^{j(r)+1}, \beta^{j(r)+2}, \ldots, \beta^{s-1}, t, \overbrace{0,0, \ldots, 0}^{u-1}<\gamma^{j(r)+1}, \gamma^{j(r)+2} \ldots, \gamma^{s-1}, \gamma^{s}
$$

or

$$
\beta^{j(r)+1}, \beta^{j(r)+2}, \ldots, \beta^{s-1},-t, \overbrace{0,0, \ldots, 0}^{u-1}<\gamma^{j(r)+1}, \gamma^{j(r)+2}, \ldots, \gamma^{s-1}, \gamma^{s}
$$


(of course, if $s=j(r)+1$ then the blocks $\beta^{j(r)+1}, \beta^{j(r)+2}, \ldots, \beta^{s-1}$ should be deleted; a similar situation also appears in some cases below). For example assume that the second possibility holds. Then define $\theta$ by $\theta^{s}=-t, \overbrace{0,0, \ldots, 0}^{u-1}$ and $\theta^{j}=\beta^{j}$ for any $j \neq s$.

Subcase 1.b. $\beta^{s} \neq \gamma^{s}$. Now we have

$$
\beta^{j(r)+1}, \beta^{j(r)+2}, \ldots, \beta^{s} \neq \gamma^{j(r)+1}, \beta^{j(r)+2}, \ldots, \gamma^{s} .
$$

Let $u>o(j(r+1)-1)$ be the first index such that $\beta_{u} \neq 0$. Then we define $\theta$ by $\theta^{j(r+1)}=\beta_{o(j(r+1)-1)+1}, \beta_{o(j(r+1)-1)+2}, \ldots,-\beta_{u}, \beta_{u+1}, \ldots, \beta_{o(j(r+1))}$ and $\theta^{j}=\beta^{j}$ otherwise.

Case 2. There are an $r>l$ and consecutive numbers $j(r)<s<v<$ $j(r+1)-1$ such that $\beta^{s}=\beta^{v}=1,0$ and $v-s$ is odd.

We can assume that $s$ and $v$ are minimal with the described properties. Say for example that $\delta_{o(j(r))}^{\beta}=-1$. Then we have either

$$
\begin{aligned}
\beta^{j(r)+1}, \beta^{j(r)+2}, \ldots, \beta^{s-1}, 1,0, \overbrace{0,0, \ldots, 0}^{t},-1,0 \\
<\gamma^{j(r)+1}, \gamma^{j(r)+2} \ldots, \gamma^{s-1}, \gamma^{s}, \gamma^{s+1}, \ldots, \gamma^{v}
\end{aligned}
$$

or

$$
\begin{aligned}
\beta^{j(r)+1}, \beta^{j(r)+2}, \ldots, \beta^{s-1},-1,0, \overbrace{0,0, \ldots, 0}^{t}, 1,0 \\
<\gamma^{j(r)+1}, \gamma^{j(r)+2} \ldots, \gamma^{s-1}, \gamma^{s}, \gamma^{s+1}, \ldots, \gamma^{v}
\end{aligned}
$$

for the even number $t=v-s-1$. Say that we are in the second case. Then define $\theta$ by $\theta^{s}=-1,0, \theta^{v}=1,0$ and $\theta^{j}=\beta^{j}$ otherwise.

Case 3. There are an $r>l$ such that $\gamma^{j(r)}=\gamma^{j(r+1)}=0$ and an $j(r)<s<j(r+1)$ such that $\gamma^{s}=1,0$ and $\beta^{s}, \beta^{s+1}, \ldots, \beta^{j(n+1)}<$ $\gamma^{s}, \gamma^{s+1}, \ldots, \gamma^{j(n+1)}$.

Define $\theta$ by $\theta^{s}=-1,0$ and $\theta^{j}=\beta^{j}$ otherwise.

Case 4. There is an $r>l$ such that $\gamma^{j(r)} \neq 0$ and $\beta^{j(r)} \neq 1,1$.

Again we must distinguish some different possibilities.

Subcase 4.a. $\beta_{o(j(r))}=0$. As in Subcase 1.b, let $u>o(j(r+1)-1)$ be the first index such that $\beta_{u} \neq 0$ and define $\theta$ by

$$
\theta^{j(r+1)}=\beta_{o(j(r+1)-1)+1}, \beta_{o(j(r+1)-1)+2}, \ldots,-\beta_{u}, \beta_{u+1}, \ldots, \beta_{o(j(r+1))}
$$

and $\theta^{j}=\beta^{j}$ otherwise. 
Subcase 4.b. $\beta_{o(j(r))} \neq 0$. In this case we shall define $\theta$ by $\theta^{j}=\beta^{j}$ for any $j \neq j(r)$, while $\theta^{j(r)}$ will be chosen in such a way that $\tau\left(\theta^{j(r)}\right)=$ $\tau\left(\beta^{j(r)}\right), \theta_{o(j(r))} \neq 0$ and either $\theta^{j(r)}<\gamma^{j(r)}$ and $\beta^{j(r)}<\gamma^{j(r)}$ or $\theta^{j(r)}>$ $\gamma^{j(r)}$ and $\beta^{j(r)}>\gamma^{j(r)}$. To define $\theta^{j(r)}$ we consider several cases.

- There is some $o(j(r)-1)<u<o(j(r))$ such that $\beta_{u} \neq 1$. Then put

$$
\theta^{j(r)}=\beta_{o(j(r)-1)+1}, \beta_{o(j(r)-1)+2}, \ldots, \beta_{o(j(r)-1},-\beta_{o(j(r))} .
$$

- $\left|\beta_{o(j(r))}\right|>1$. Then put

$$
\begin{aligned}
& \theta^{j(r)}=-\left|\beta_{o(j(r)-1)+1}\right|-2^{o(j(r))-o(j(r)-1)-1}, \\
& \quad \beta_{o(j(r)-1)+2}, \ldots, \beta_{o(j(r))-1},\left|\beta_{o(j(r))}\right|-1 \\
& \text { if } \beta^{j(r)<\gamma^{j(r)} \text { and }} \\
& \theta^{j(r)}=\left|\beta_{o(j(r)-1)+1}\right|+2^{o(j(r))-o(j(r)-1)-1}, \\
& \beta_{o(j(r)-1)+2}, \ldots, \beta_{o(j(r))-1},\left|\beta_{o(j(r))}\right|-1
\end{aligned}
$$

if $\beta^{j(r)}>\gamma^{j(r)}$.

- $\beta^{j(r)}=1,1, \ldots, 1,-1$. Then put $\theta^{j(r)}=-1,1, \ldots, 1,1$.

- $\beta^{j(r)}=1,1,1 \ldots, 1,1$. Then put $\theta^{j(r)}=3,0,1, \ldots, 1,1$ (here recall that $\left.\beta^{j(r)} \neq 1,1\right)$.

Case 5. There are an $r>l$ such that $\beta^{j(r+1)}=1,1$ and an $j(r)<s<$ $j(r+1)$ such that $\beta^{s}=1,0$ and $j(r+1)-s$ is odd.

Define $\theta_{s}=-1,0$ and $\theta^{j}=\beta^{j}$ otherwise.

Case 6. There is an $r>l$ such that $\beta^{j(r)}=1,1$ and $\delta_{o(j(r))}^{\beta}=1$.

Then define $\theta^{j(r)}=3,0$ and $\theta^{j}=\beta^{j}$ otherwise.

Case 7. There are an $r>l$ such that $\beta^{j(r)}=1,1$ and $\delta_{o(j(r)}^{\beta}=-1$ and an $j(r)<s<j(r+1)$ such that $\beta^{s}=1,0$ and $s-j(r)$ is odd.

Define $\theta_{s}=-1,0$ and $\theta^{j}=\beta^{j}$ otherwise.

This finishes the proof of the lemma.

Proof of Theorem 2.5: It follows from Lemmas 3.1, 3.2 and 3.3.

\section{References}

1. V. JimÉnEz LóPEz, An explicit description of all scrambled sets of weakly unimodal functions of type $2^{\infty}$, Real Anal. Exchange, to appear. 
2. M. Kuchta And J. Smital, Two point scrambled set implies chaos (Proceedings of the European Conference on Iteration Theory, ECIT 87), (Caldes de Malavella, Spain, 1987), World Sci. Publishing, Singapore, 1989, pp. 427-430.

3. T. Y. Li And J. A. Yorke, Period three implies chaos, Amer. Math. Monthly 82 (1975), 985-992.

4. J. Milnor And W. Thurston, On iterated maps of the interval, in "Dynamical Systems," Springer, Berlin, 1988, pp. 465-563.

5. M. Misiurewicz And J. Smital, Smooth chaotic functions with zero topological entropy, Ergodic Theory Dynam. Systems 8 (1988), 421-424.

6. C. Preston, "Iterates of Maps on an Interval," Lecture Notes in Mathematics 999, Springer, Berlin, 1983.

7. J. Smital, Chaotic functions with zero topological entropy, Trans. Amer. Math. Soc. 297 (1986), 269-282.

8. S. J. VAn Strien, Smooth dynamics on the interval, in "New Directions in Dynamical Systems," T. Bedford and J. Swift, eds., Cambridge Univ. Press, 1988, pp. 57-119.

1991 Mathematics subject classifications: Primary, 58F13. Secondary, 26A18, 54H20

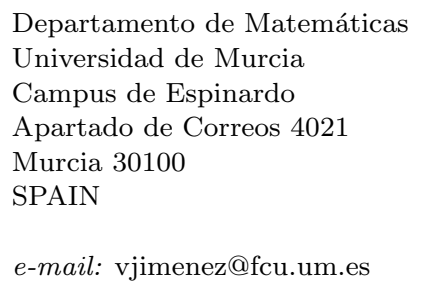

Primera versió rebuda el 31 de Juliol de 1995, darrera versió rebuda el 26 de Febrer de 1996 